\title{
Correction to: Hydrothermally induced changes in the properties of MFC and characterization of the low molar mass degradation products
}

\author{
Salla Hiltunen (D) - Krista Koljonen • Klaus Niemelä • Isto Heiskanen • \\ Leena-Sisko Johansson • Kaj Backfolk
}

Published online: 6 September 2019

(C) The Author(s) 2019

\section{Correction to: Cellulose}

https://doi.org/10.1007/s10570-019-02603-w

In the original publication, the less than symbol $(<)$ was mistakenly processed as greater than symbol $(>)$ in Tables 2 and 4 . Also in the caption of these tables, the amount of detected products is reported per gram of raw material. The correct version of Tables 2 and 4 are provided below.

The original article can be found online at https://doi.org/10.1007/s10570-019-02603-w.

S. Hiltunen $(\bowtie) \cdot K$. Koljonen · K. Backfolk

LUT University, Yliopistonkatu 34, 53850 Lappeenranta, Finland

e-mail: salla.hiltunen@lut.fi

K. Niemelä

VTT Technical Research Centre of Finland, Tietotie 2,

02150 Espoo, Finland

I. Heiskanen · K. Backfolk

Research Centre Imatra, Stora Enso Oyj, 55800 Imatra,

Finland

L.-S. Johansson

Aalto University, Vuorimiehentie 1, 000760 Aalto,

Finland 
Table 2 The main sugars, acids and furan compounds found in the filtrates $\left(\mathrm{mg} / \mathrm{g}_{M F C}\right)$ n.a. not analyzed, tr. traces, empty cells indicate not reliably detected

\begin{tabular}{|c|c|c|c|c|c|c|}
\hline \multirow[t]{2}{*}{ Compound } & \multicolumn{6}{|c|}{ Treatment time (h) at $150^{\circ} \mathrm{C}$} \\
\hline & 0 & 0.5 & 2.5 & 4.5 & 8.5 & 20.5 \\
\hline Xylose & $<0.5$ & 0.5 & 2.3 & 3.8 & 8.1 & 57.5 \\
\hline Xylulose & & & & tr. & $<0.5$ & 1 \\
\hline Glucose & & $<0.5$ & $<0.5$ & 0.5 & 0.5 & 3.1 \\
\hline Fructose & & & & & tr. & $<0.5$ \\
\hline Other monosaccharides & & & $<0.5$ & $<0.5$ & $<0.5$ & 1.2 \\
\hline Carbohydrate-type unknowns & & & & tr. & $<0.5$ & 1 \\
\hline Xylobiose & & $<0.5$ & 0.8 & 1.4 & 3.6 & 12 \\
\hline Xylotriose & & tr. & $<0.5$ & $<0.5$ & 0.9 & 1.4 \\
\hline Cellobiose & $<0.5$ & $<0.5$ & 0.5 & 0.6 & $<0.5$ & 1.4 \\
\hline Formic acid & & 1.1 & 1.1 & 1.4 & 1.6 & 2.4 \\
\hline Acetic acid & $<0.5$ & $<0.5$ & $<0.5$ & $<0.5$ & 0.5 & 0.7 \\
\hline Furfural & $<5$ & n.a. & n.a. & n.a. & $<5$ & 10.3 \\
\hline 5-Hydroxymethylfurfural & $<5$ & n.a. & n.a. & n.a. & $<5$ & $<5$ \\
\hline Glycolic acid & & $<0.5$ & 0.5 & 0.5 & $<0.5$ & 1.3 \\
\hline Oxalic acid & & & & & tr. & $<0.5$ \\
\hline Pyruvic acid & & & & & & $<0.5$ \\
\hline Lactic acid & & & $<0.5$ & $<0.5$ & $<0.5$ & $<0.5$ \\
\hline Glyceric acid & & & $<0.5$ & $<0.5$ & $<0.5$ & $<0.5$ \\
\hline 2-Hydroxybutanoic acid & & & & tr. & tr. & $<0.5$ \\
\hline 2,4-Dihydroxybutanoic acid & & & $<0.5$ & $<0.5$ & $<0.5$ & $<0.5$ \\
\hline Threonic acid & & & $<0.5$ & $<0.5$ & tr. & $<0.5$ \\
\hline 3-Deoxypentonic acid (2 isomers) & & & $<0.5$ & $<0.5$ & $<0.5$ & 1.4 \\
\hline 3-Deoxyhexonic acid (2 isomers) & & & $<0.5$ & $<0.5$ & tr. & 0.6 \\
\hline Xylonic acid & $<0.5$ & $<0.5$ & $<0.5$ & $<0.5$ & $<0.5$ & 0.8 \\
\hline
\end{tabular}


Table 4 Products detected $\left(\mathrm{mg} / \mathrm{g}_{\text {sugar }}\right)$ after hydrothermal treatment of xylose and glucose at $150{ }^{\circ} \mathrm{C}$ for $20.5 \mathrm{~h}$

\begin{tabular}{|c|c|c|}
\hline Compound or compound category & Xylose & Glucose \\
\hline Glycolic acid & 1.8 & 1.1 \\
\hline Oxalic acid & $<0.5$ & $<0.5$ \\
\hline Lactic acid & 0.5 & $<0.5$ \\
\hline Pyruvic acid & tr. & tr. \\
\hline 3-Hydroxypropanoic acid & 0.5 & $<0.5$ \\
\hline Glyceric acid & 0.5 & $<0.5$ \\
\hline 2,4-Dihydroxybutanoic acid & $<0.5$ & $<0.5$ \\
\hline Erythronic acid & $<0.5$ & tr. \\
\hline Threonic acid & $<0.5$ & tr. \\
\hline Levulinic acid & n.d. & 0.4 \\
\hline 2-Oxoglutaric acid & n.d. & tr. \\
\hline 3-Deoxy-erythro-pentonic acid & 1.2 & n.d. \\
\hline 3-Deoxy-threo-pentonic acid & 2.9 & n.d. \\
\hline Xylonic acid & $\operatorname{tr}$ & n.d. \\
\hline 3-Deoxyhexonic acid (3-4 isomers) & n.d. & 5.7 \\
\hline Gluconic acid & n.d. & 1.2 \\
\hline Tetroses & tr. & tr. \\
\hline Xylulose & 7 & 0 \\
\hline Other pentoses & 7.1 & 0.6 \\
\hline Fructose & n.d. & 19.8 \\
\hline Other hexoses & 0.7 & 1 \\
\hline Unknown sugar-type compounds & 3.9 & 6.6 \\
\hline Unknown alditol-type compounds & 4.7 & 1.1 \\
\hline Disaccharide-type compounds & 0.6 & 3 \\
\hline Unusual polyhydroxy compounds & 0.9 & 12.7 \\
\hline Reductic acid & 1.4 & n.d. \\
\hline 5-Hydroxymethylfurfural & n.d. & 32.8 \\
\hline 2-Furancarboxylic acid & tr. & tr. \\
\hline Catechol & tr. & tr. \\
\hline Hydroquinone & n.d. & tr. \\
\hline Methylcatechol(s) & tr. & tr. \\
\hline 3,4-Dihydroxybenzaldehyde & $<0.5$ & n.d. \\
\hline Pyrogallol (1,2,3-Benzenetriol) & n.d. & $<0.5$ \\
\hline 1,2,4-Benzenetriol & n.d. & 2.7 \\
\hline Benzenetetrol & n.d. & $<0.5$ \\
\hline 3,8-Dihydroxy-2-methylchromone & $\operatorname{tr}$ & n.d. \\
\hline Unknown aromatic/phenolic compounds & 0.5 & $<0.5$ \\
\hline
\end{tabular}

n.d. not detected, $t r$. traces
Open Access This article is distributed under the terms of the Creative Commons Attribution 4.0 International License (http://creativecommons.org/licenses/by/4.0/), which permits unrestricted use, distribution, and reproduction in any medium, provided you give appropriate credit to the original author(s) and the source, provide a link to the Creative Commons license, and indicate if changes were made.

Publisher's Note Springer Nature remains neutral with regard to jurisdictional claims in published maps and institutional affiliations. 